\title{
Comet composition and Lab
}

\author{
Dominique Bockelée-Morvan and Nicolas Biver \\ LESIA, Observatoire de Paris, LESIA/CNRS, UPMC, Université Paris-Diderot, \\ F-92195 Meudon, France \\ email: dominique.bockelee@obspm.fr
}

\begin{abstract}
Comet composition and properties provide information on chemical and physical processes that occurred in the early Solar system, 4.6 Gyr ago. The study of comets and of starforming regions both help for a better understanding of the formation of planetary systems. A review of our present knowledge of cometary composition is presented. We also discuss laboratory studies that would be helpful for data analysis.
\end{abstract}

Keywords. comets: general, solar system: formation

\section{Introduction}

The solar system formed about 4.6 billion years ago from an infall of matter inside a molecular cloud. A wealth of processes happened, involving chemistry, and dynamics at all scales before the solar system reached its present state with its cortege of planets and small bodies. Comets formed in the outer skirts of the solar nebula and trapped volatile species formed either in the presolar cloud or in situ. Hence, the study of the composition of comets provides insights on the formation of the solar system, and protoplanetary disks, in general.

\section{Comet composition from spectroscopy}

The composition of cometary ices is best studied from observations of cometary atmospheres. At the present date, 28 molecules (not including isotopologues) have been identified by spectroscopy in the UV to the millimeter and submillimeter domains (Fig. 1). For a recent overview, see Cochran et al. (2015). The most recent discoveries are glycolaldehyde $\left(\mathrm{CH}_{2} \mathrm{OHCHO}\right)$ and ethanol $\left(\mathrm{C}_{2} \mathrm{H}_{5} \mathrm{OH}\right)$, identified in comet $\mathrm{C} / 2014$ Q2 (Lovejoy) from lines in the millimeter range (Biver et al. 2015). Whereas most molecules are observed at millimeter wavelengths, the infrared domain gives access to molecules with no dipole moments as $\mathrm{CO}_{2}, \mathrm{CH}_{4}$, etc.

It is striking that the molecular complexity observed in comets resembles that observed in star-forming regions. A comparison between the abundances of complex organics observed in comets to values measured around high-mass and low mass proto-stars shows strong similarities, in line with their synthesis through grain-surface reactions in the presolar cloud or in the solar nebula (Biver et al. 2015).

\section{Laboratory requests}

Further progress in the characterization of cometary volatiles through infrared and mm-wave spectroscopy is expected in the near future, e.g., with ALMA and 30-m class optical telescopes. The following laboratory data and/or theoretical studies would be helpful for the analysis of cometary spectra: 


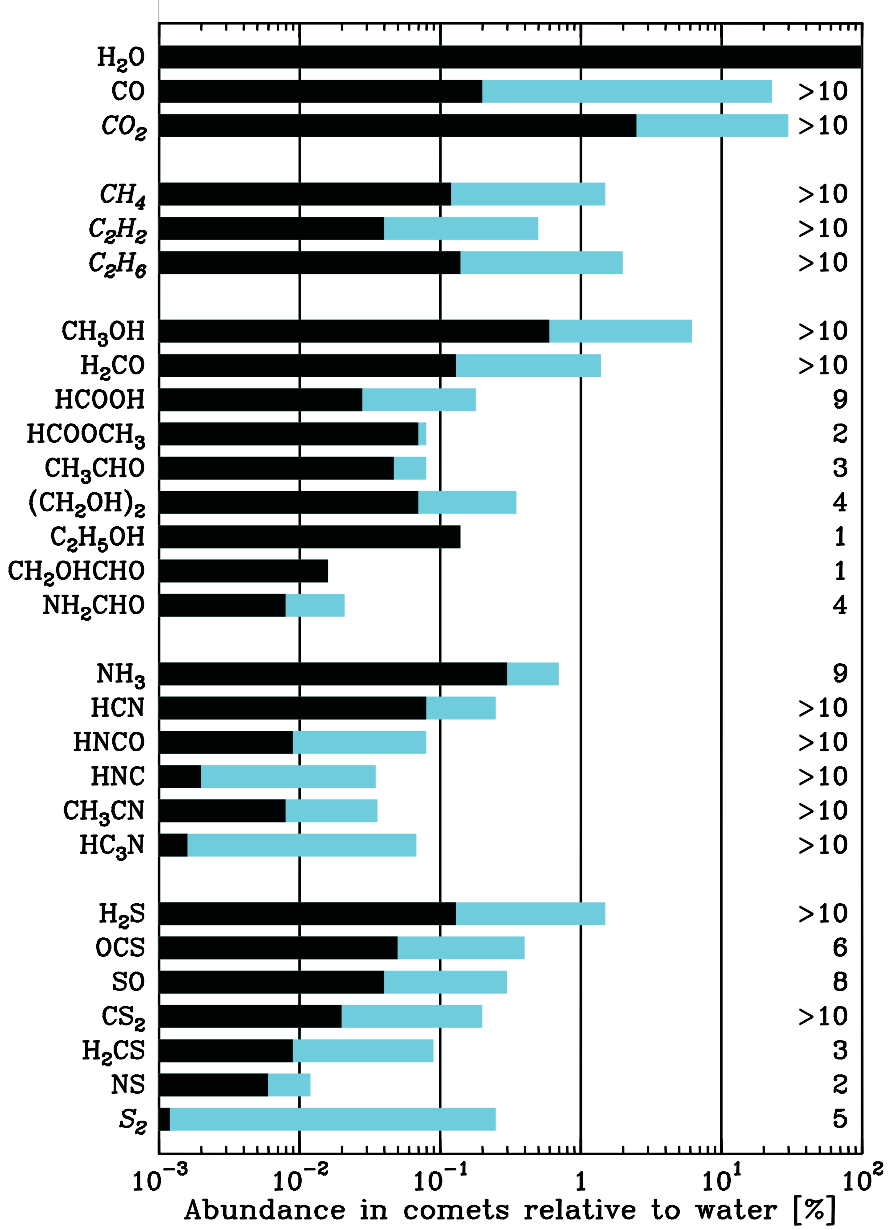

Figure 1. Molecules detected in comets and their abundances relative to water. Bars in blue (grey) show the range of measured abundances in comets, indicating composition diversity between comets. The number of comets in which abundance measurements are available is indicated in the right. This figure is an updated version of that published in Bockelée-Morvan et al. (2004)

- Rotational lines: lines frequencies and strengths for complex species. Molecules present in cometary atmospheres are similar to those found in molecular hot-cores and hot-corinos. Therefore, cometary science will benefit from spectral characterizations performed for assigning unidentified lines in ISM spectra. Specific requests may follow identifications made from the Rosina mass spectrometer onboard Rosetta (Balsiger et al. 2007).

- Rotational lines: In cometary atmospheres, the collisional partner is $\mathrm{H}_{2} \mathrm{O}$. Collisional cross-sections involving water are not available for most molecules, whereas they are an important parameter for determining accurate column densities from observed line intensities. Collisional cross-sections in the temperature range 20-120 K are needed.

- Rotational lines: Besides collisional excitation, rotational levels in the ground vibrational state are pumped through IR excitation of the vibrational bands by solar 


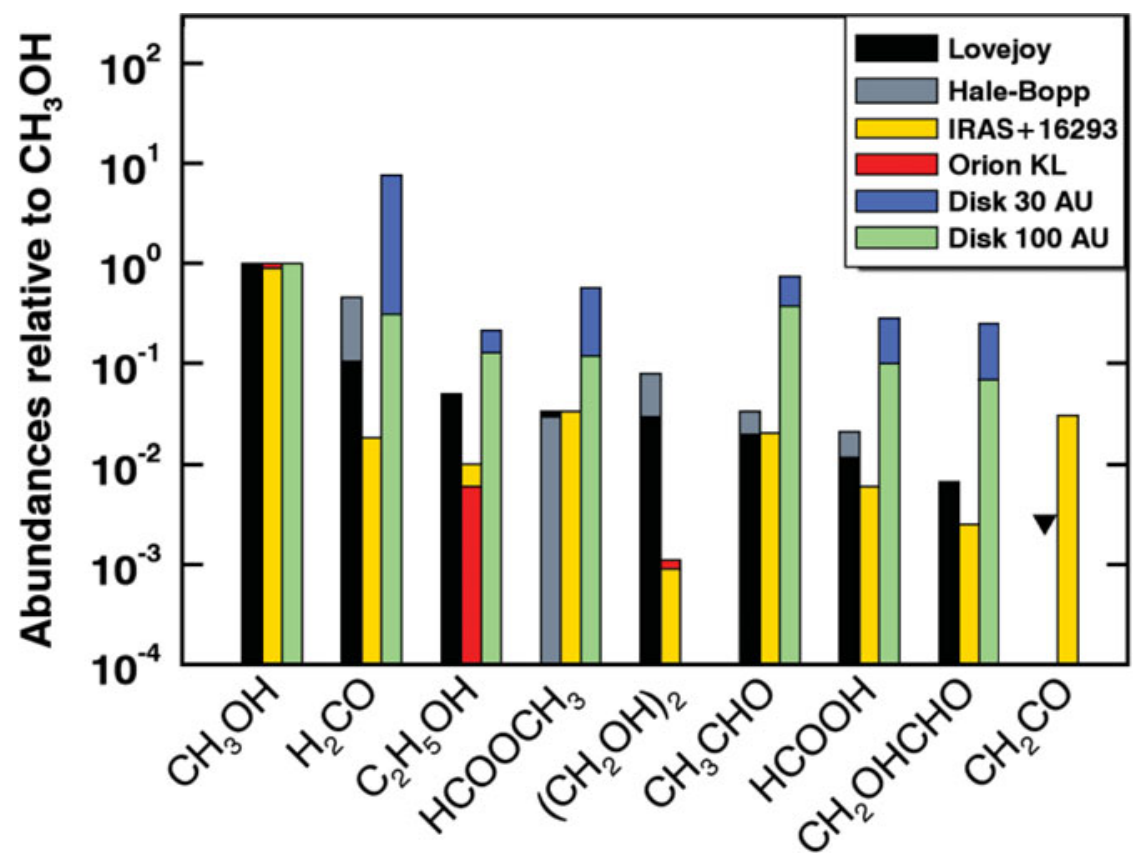

Figure 2. Abundances of complex organics in comets, molecular hot-core (Orion KL) and hot-corinos (IRAS+162293). Results from chemical modelling of protoplanetary disks (Walsh et al. (2014)) are also plotted. Figure from Biver et al. (2015).

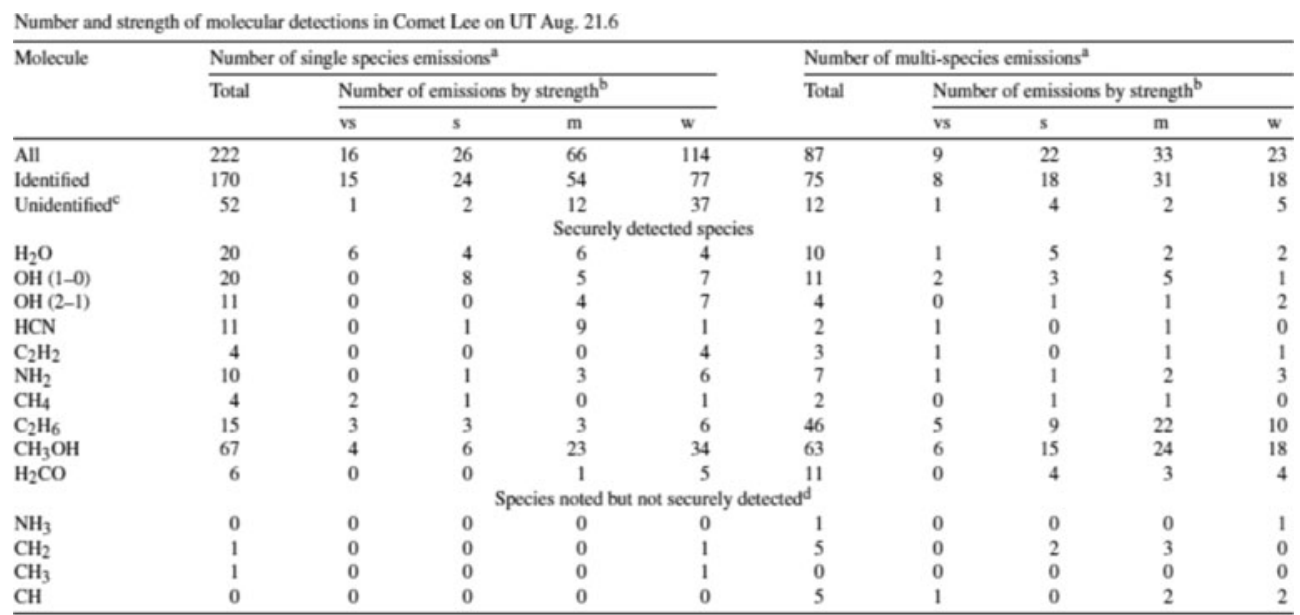

Figure 3. Infrared spectral survey of comet C/1999 H1 (Lee) covering the 3479-2702 $\mathrm{cm}^{-1}$ domain (extracted from Dello Russo et al. 2006). Strength: $v s=$ very strong, $s=$ strong, $m=$ medium; $w=$ weak

radiation. This fluorescent excitation cannot be computed for several detected molecules (e.g., $\mathrm{HNCO}, \mathrm{HCOOH}$, and complex organics) because information is missing, implying calculations in the LTE approximation.

- Vibrational bands: line-by-line ro-vibrational strengths and frequencies of many simple and complex CHO-bearing molecules are not available in the literature. This is the case for $\mathrm{CH}_{3} \mathrm{OH}$, the main $\mathrm{CHO}$ molecules in cometary atmospheres, which presents many strong ro-vibrational lines in the 3.3-3.5 $\mu \mathrm{m}$ domain (e.g., Villanueva et al. 2012, 
Disanti et al. 2013). In addition, cometary spectra observed with high spectral resolution present many unidentified lines in this spectral region (e.g., Dello Russo et al. 2006, Dello Russo et al. 2013). In the spectral survey of comet C/1999 H1 (Lee) covering the 3479$2702 \mathrm{~cm}^{-1}$ domain, 101 out of 545 lines remain partially or completely unidentified, with the strongest lines in the $3300-3470 \mathrm{~cm}^{-1}$ range (Fig. 2, Dello Russo et al. 2006).

- Molecular photodissociation rates: Under the influence of solar radiation, molecules photodissociate in cometary atmospheres. Photodissociation rates are important parameters for the determination of molecular production rates. Compilations of determined values can be found in Huebner et al. (1992) and Crovisier (1994). Values are missing for several detected molecules. Photodissociation channels and quantum yields are also important quantities to investigate production mechanisms of detected molecules (Crovisier 1994).

\section{Conclusion}

In this paper, we focussed on laboratory data that would be helpful for analysing gasphase signatures observed in the infrared and microwave domains. The optical domain, where many lines remain also unidentified in cometary spectra, would also benefit from laboratory investigations (Cochran \& Cochran 2002). There are many other domains where laboratory experiments support cometary science, as illustrated in Gudipati et al. (2015). One can anticipate that the wealth of data acquired by the instruments onboard the Rosetta spacecraft will initiate many laboratory studies.

\section{References}

Balsiger, H., Altwegg, K., Bochsler, P., et al. 2007, Space Science Reviews, 128, 745 Biver, N., Bockelée-Morvan, D., Moreno, R., et al. 2015, Science Advances, in press Bockelée-Morvan, D., Crovisier, J., Mumma, M. J., \& Weaver, H. A. 2004, Comets II, 391 Cochran, A. L. \& Cochran, W. D. 2002, Icarus, 157, 297

Cochran, A. L., Levasseur-Regourd, A.-C., Cordiner, M., et al. 2015, Space Science Reviews, 68 Crovisier, J. 1994, JGR, 99, 3777

Dello Russo, N., Mumma, M. J., DiSanti, M. A., et al. 2006, Icarus, 184, 255

Dello Russo, N., Vervack, R. J., Weaver, H. A., et al. 2013, Icarus, 222, 707

DiSanti, M. A., Bonev, B. P., Villanueva, G. L., \& Mumma, M. J. 2013, ApJ, 763, 1

Huebner, W. F., Keady, J. J., \& Lyon, S. P. 1992, Astrophys. Space Sci., 195, 1-294, 1992.

Gudipati, M. S., Abou Mrad, N., Blum, J., et al. 2015, Space Science Reviews, 79

Villanueva, G. L., DiSanti, M. A., Mumma, M. J., \& Xu, L.-H. 2012, ApJ, 747, 37

Walsh, C., Millar, T. J., Nomura, H., et al. 2014, A\&A, 563, A33 